\section{REFERENCES}

1 Galie N, Hoeper MM, Humbert M, et al. Guidelines for the diagnosis and treatment of pulmonary hypertension. Eur Respir J 2009; 34: 1219-1263.

2 Somlyo AP, Somlyo AV. Signal transduction by G-proteins, rhokinase and protein phosphatase to smooth muscle and non-muscle myosin II. J Physiol 2000; 522: 177-185.

3 Etienne-Manneville S, Hall A. Rho GTPases in cell biology. Nature 2002; 420: 629-635.

4 Abe K, Shimokawa H, Morikawa K, et al. Long-term treatment with a Rho-kinase inhibitor improves monocrotaline-induced fatal pulmonary hypertension in rats. Circ Res 2004; 94: 385-393.

5 Dahal BK, Kosanovic D, Pamarthi PK, et al. Therapeutic efficacy of azaindole-1 in experimental pulmonary hypertension. Eur Respir J 2010; 36: 808-818.

6 Mouchaers KT, Schalij I, de Boer MA, et al. Fasudil reduces monocrotaline-induced pulmonary arterial hypertension: comparison with bosentan and sildenafil. Eur Respir J 2010; 36: 800-807.
7 Oka M, Homma N, Taraseviciene-Stewart L, et al. Rho kinasemediated vasoconstriction is important in severe occlusive pulmonary arterial hypertension in rats. Circ Res 2007; 100: 923-929.

8 Fukumoto Y, Matoba T, Ito A, et al. Acute vasodilator effects of a Rho-kinase inhibitor, fasudil, in patients with severe pulmonary hypertension. Heart 2005; 91: 391-392.

9 Ishikura K, Yamada N, Ito M, et al. Beneficial acute effects of rhokinase inhibitor in patients with pulmonary arterial hypertension. Circ J 2006; 70: 174-178.

10 Abbas AE, Fortuin FD, Schiller NB, et al. A simple method for noninvasive estimation of pulmonary vascular resistance. $J \mathrm{Am}$ Coll Cardiol 2003; 41: 1021-1027.

11 Fujita H, Fukumoto Y, Saji K, et al. Acute vasodilator effects of inhaled fasudil, a specific Rho-kinase inhibitor, in patients with pulmonary arterial hypertension. Heart Vessels 2010; 25: 144-149.

\title{
Nuclear magnetic resonance-based metabolomics of exhaled breath condensate: methodological aspects
}

\section{To the Editors:}

Due to the lack of standardised procedures for exhaled breath condensate (EBC), a noninvasive technique for investigating lung inflammatory mediators [1], the between-laboratory comparison of results is difficult. Moreover, different collecting devices have been reported to influence the EBC content [2,3].

The analysis of metabolic profiles ("metabolomics") of EBC using nuclear magnetic resonance (NMR) spectroscopy discriminates between chronic obstructive pulmonary disease (COPD) patients and healthy subjects (HS) [4]; asthmatic children and HS [5]; and patients with stable cystic fibrosis and unstable cystic fibrosis and HS [6].

This approach has recently been questioned as NMR-based metabolomics of EBC collected using a condenser with reusable parts was reported to be affected by cleaning procedures, generating artificial signals that were not related to the endogenous metabolites of the lungs [7].

In this study we assessed the effects of a different cleaning procedure of a reusable-part condenser on EBC metabolomics; the possible time and carry-over effects when the same device is repeatedly used; technique sensitivity; the ability of NMR spectroscopy of EBC to discriminate between COPD patients and HS; and the potential of NMR spectroscopy in identifying selective EBC metabolites.

If the cleaning procedure produces artificial signals in the NMR spectra of EBC, the separation between COPD patients and HS reported previously [4] is certainly surprising, as the residual signals derived from the disinfectant Descogen (Antiseptica chem.-pharm. Produkte GmbH, Pulheim/Brauweiler, Germnay) should have randomly affected both groups. Moreover, using a different reusable-part condenser, CARRARO et al. [5] reported that
NMR-based metabolomics of EBC differentiates asthmatic children from HS with a success rate of $86 \%$ [5].

To verify the influence of the disinfectant on EBC metabolomics, we modified the proposed cleaning procedure [4] by replacing Descogen with Milton (Milton Pharmaceutical UK Ltd, Gloucester, UK), a pure sodium hypochlorite solution. The reusable parts of the EcoScreen condenser (Jaeger, Hoechberg, Germany) were soaked for 15 min with a $3.55 \mathrm{mM}$ Milton solution (according to manufacturer guidelines), and then flushed for $15 \mathrm{~min}$ with deionised distilled water. ${ }^{1} \mathrm{H}-\mathrm{NMR}$ spectra were recorded at $600 \mathrm{MHz}(14.1 \mathrm{~T})$ on a Bruker Avance spectrometer (Bruker BioSpin $\mathrm{GmbH}$, Rheinstetten, Germany) equipped with a 5-mm CPTCI CryoProbe ${ }^{\mathrm{TM}}$ (Bruker BioSpin GmbH).

Figure 1a reports the NMR spectrum of the Milton solution. Except for the reference $0.1 \mathrm{mM}$ sodium 3-trimethylsilyl $\left[2,2,3,3-{ }^{2} \mathrm{H}_{4}\right]$ propionate (TSP) signal at $0.0 \mathrm{ppm}$, no other signals were present in the spectrum. The absence of signals (implying the absence of contaminant) was confirmed in the vertical expansion of the spectrum (fig. 1b). To exclude the presence of contaminants in the deionised water used for rinsing the collection set-up, we acquired the spectrum of the soaking water after rinsing for $5 \mathrm{~min}$ (fig. 1c). No peak was observed, indicating that the disinfection and the rinsing steps of the EBC collection set-up do not affect the EBC spectra. These data exclude the fact that cleaning procedure based on Milton solution affects NMR spectroscopy of EBC, indicating that the observed profiles of EBC metabolites are not artificial signals but represent endogenous metabolites.

When a reusable-part condenser (EcoScreen) was repeatedly used [8], the measurement of several EBC biomarkers detected no carry-over effects from previous sampling periods. If carry-over effects were present, the metabolites would display an "average 
a)

b)

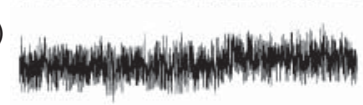

c)

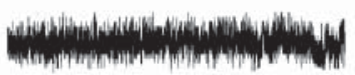

d)

e)
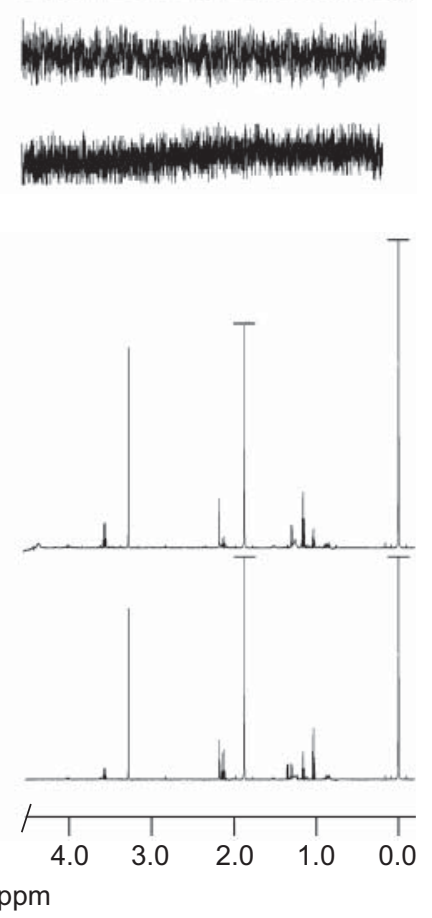

FIGURE 1. ${ }^{1} \mathrm{H}$-nuclear magnetic resonance spectra of sodium hypochlorite cleaning solution (Milton; Milton Pharmaceutical UK Ltd, Gloucester, UK), which is used for the reusable parts of an EcoScreen condenser, and exhaled breath condensate (EBC) samples. a) A $3.55 \mathrm{mM}$ Milton solution (512 scans). Except for the reference $0.1 \mathrm{mM}$ sodium 3 -trimethylsilyl[2,2,3,3-$\left.{ }^{2} \mathrm{H}_{4}\right]$ propionate signal at $0.0 \mathrm{ppm}$, no other signals were present in the spectrum. b) The absence of contaminant peaks was confirmed in the vertical expansion $(\times 32)$ of the spectrum and c) in the deionised water used for rinsing the condenser for 5 min (1,024 scans, $\times 32$ ). Therefore, the EBC spectra (512 scans) of d) a healthy subject and e) a chronic obstructive pulmonary disease patient are not contaminated by the cleaning solution. Spectra were recorded at $300 \mathrm{~K}$.

distribution" after a number of EBC samples had been collected. The NMR spectra of EBC samples from different classes of subjects would be expected to be very similar, therefore, preventing any classification with multivariate analysis.

To verify this, 54 EBC samples were collected from 27 patients with COPD (16 males and 11 females, mean \pm SD age $58.9 \pm$ $5.7 \mathrm{yrs}$ ) and $27 \mathrm{HS}$ (16 males and 11 females, mean \pm SD age $55.6 \pm 6.2 \mathrm{yrs}$ ) using an EcoScreen condenser as described previously [4], and spectra were acquired in a randomised sequential order. The Milton cleaning procedure was applied. EBC samples were treated and stored as reported previously [4]. Sample drying was avoided to prevent irreversible solute precipitation and/or formation of insoluble aggregates, which we observed upon dissolving the dried condensate.

Figure 1d shows the EBC spectrum of a HS. Signals were identified through two-dimensional NMR experiments (data not shown), and confirmed those reported previously [4]. Compared to a HS (fig. 1d), the corresponding spectrum from a COPD patient (fig. 1e) showed some differences located in the regions centred at 2.2 and $1.2 \mathrm{ppm}$. These regions contain the propionate $\alpha \mathrm{CH}_{2}$ at $2.19 \mathrm{ppm}$ (fig. $1 \mathrm{~d}$ and e), the acetoine $\alpha \mathrm{CH}_{3}$ at $2.23 \mathrm{ppm}$ (fig. 1d), the fatty acid $\left(\mathrm{CH}_{2}\right)_{2} \mathrm{COO}$ at $2.23 \mathrm{ppm}$ (fig. 1e), the propionate $\mathrm{BCH}_{3}$ at $1.06 \mathrm{ppm}$ (fig. $1 \mathrm{~d}$ and e), the ethanol $\mathrm{CH}_{3}$ at $1.21 \mathrm{ppm}$ (fig. $1 \mathrm{~d}$ and e), the fatty acid $\mathrm{CH}_{2} \mathrm{CH}_{3}$ at $1.27 \mathrm{ppm}$ (fig. $1 \mathrm{~d}$ and e), the lactate $\mathrm{\beta CH}_{3}$ at 1.33 ppm (fig. $1 \mathrm{~d}$ and e), and the threonine $\gamma \mathrm{CH}_{3}$ at $1.39 \mathrm{ppm}$ (fig. 1e). Many other resonances of lower intensity are observed at increased vertical scale, and they have all been assigned by two-dimensional NMR experiments. The detection limit of our NMR technique was calculated by integrating $10 \mathrm{EBC}$ spectra; they were normalised to the standard TSP $(0.1 \mathrm{mM})$, obtaining an average concentration of $0.14 \pm 0.04 \mu \mathrm{M}$ for the endogenous phenylalanine (signals at 7.3$7.0 \mathrm{ppm})$, among the lowest detected signals.

Spectral differences between COPD and HS were verified by principal components analysis and partial least squares discriminant analysis. NMR-based metabolomics of EBC clearly discriminates between COPD patients and HS ( $\mathrm{r}^{2}=99.9 \%$ ) (fig. 2 ) confirming our previous NMR results on EBC collected with

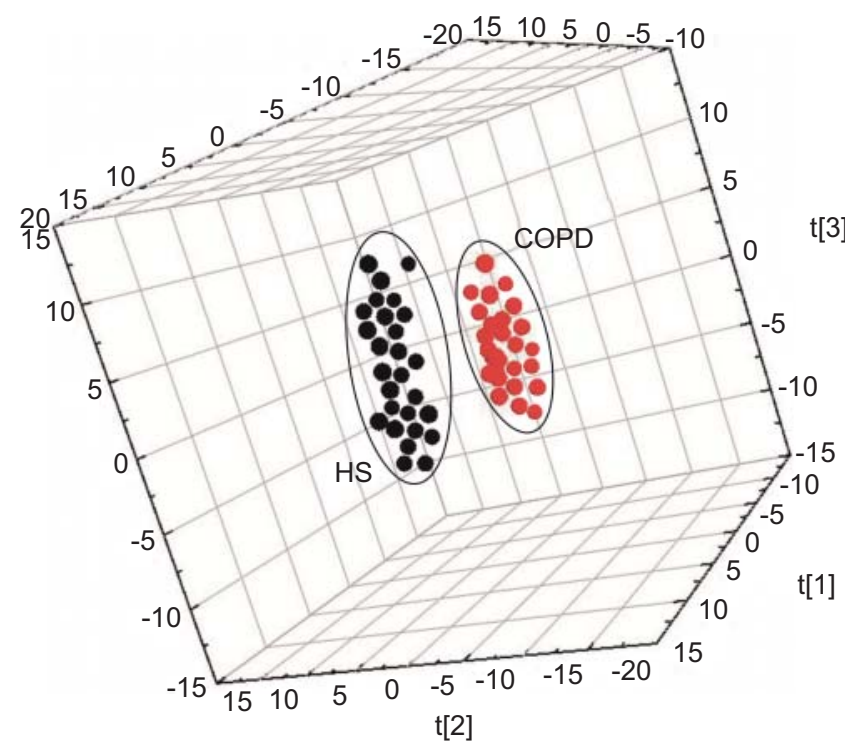

FIGURE 2. Three-dimensional score plot obtained from a partial least squares discriminant analysis based on nuclear magnetic resonance spectra of exhaled breath condensate $(\mathrm{EBC})$ from 27 patients with chronic obstructive pulmonary disease (COPD) and 27 healthy subjects (HS). All HS were nonsmokers or exsmokers. COPD patients were ex-smokers and they had stopped smoking for $\geqslant 24$ months. Diagnosis of COPD was based on the Global Initiative for Chronic Obstructive Lung Disease guidelines. No subject was exposed to organic solvents. None of the COPD patients was on systemic or inhaled corticosteroid treatment for $\geqslant 4$ weeks, and they were asked not to use long-acting $\beta_{2}$-agonist and anticholinergic agents for $\geqslant 12$ and $24 \mathrm{~h}$, respectively. No subject had COPD exacerbations or upper and/or lower airway infection in the previous 4 weeks. Subjects refrained from food intake for at least $4 \mathrm{~h}$ and from alcoholic drinks for at least $18 \mathrm{~h}$ before EBC collection. In the score plot, t[1], t[2] and t[3] represent the scores (the first three partial least squares components) of the model, which are sufficient to build a satisfactory classification model. COPD and HS EBC classes show a clear separation in two distinct clusters. The total significance of the model was $r^{2}=99.9 \%$. 
reusable-part condenser [4], and results obtained by others using a non-reusable-part condenser and mass spectrometry (MS) [7].

The data mentioned previously demonstrate that the cleaning procedure with Milton does not alter the metabolic profiles of $\mathrm{EBC}$, and that NMR spectroscopy is suitable for investigating EBC samples.

By using an Anacon condenser (Biostec, Valencia, Spain), different results have been reported [7]. Collection devices are an important source of variability of EBC biomarkers [2, 3, 8]. The principal variability factors include cooling temperature [9] and condenser materials [2]. For example, a warm-up during condensation is observed when RTube or Anacon are used (condensers using a disposable collection kit), whereas EcoScreen cools down slightly during the procedure [8,9]. Such differences affect biomarker concentrations $[2,9]$. More importantly, there was no correlation between biomarkers measured in EBC collected with EcoScreen and Anacon condensers [9], and there was only an $\sim 60 \%$ correlation between biomarkers measured in EBC collected with RTube and Anacon condensers; although both used a disposable collection kit [9]. However, as no NMR details were found (i.e. the operating magnetic field, the number of acquisitions, the possible use of a cryoprobe, and the detection limit), it is difficult to conclude that NMR spectroscopy cannot be used to analyse EBC samples because it does not have the sensitivity required to observe the endogenous metabolites in the EBC [7]. With our spectrometer set-up, we have estimated a detection limit that is notably low for NMR-based metabolomics [10].

In conclusion, our cleaning procedure of EBC collection set-up does not generate artificial signals in the metabolic profile of EBC. Furthermore, NMR-based metabolomics are suitable for identifying specific EBC metabolites and are potentially useful for characterising the metabolic fingerprints of patients with respiratory diseases. Although a head-to-head comparison of different condensers is required, the combination of different reference analytical techniques, including NMR and MS, might consolidate "breathomics" as a new noninvasive approach to the assessment of patients with respiratory disease with important diagnostic and therapeutic implications.

A. Motta*, D. Paris*, D. Melck*, G. de Laurentiis", M. Maniscalco\#, M. Sofia" and P. Montuschi ${ }^{*}$

*Institute of Biomolecular Chemistry, National Research Council, Pozzuoli, "Dept of Respiratory Medicine, A.O. Monaldi,
University of Naples "Federico II", Naples, and "Dept of Pharmacology, Faculty of Medicine, Catholic University of the Sacred Heart, Rome, Italy.

Correspondence: P. Montuschi, Dept of Pharmacology, Faculty of Medicine, Catholic University of the Sacred Heart, Largo Francesco Vito 1, I-00168 Rome, Italy. E-mail. pmontuschi@rm. unicatt.it

Statement of Interest: None declared.

\section{REFERENCES}

1 Horvath I, Hunt J, Barnes PJ. Exhaled breath condensate: methodological recommendations and unresolved questions. Eur Respir J 2005; 26: 523-548.

2 Rosias P, Robroeks C, Kester A, et al. Biomarker reproducibility in exhaled breath condensate collected with different condensers. Eur Respir J 2008; 31: 934-942.

3 Koczulla R, Dragonieri S, Schot R, et al. Comparison of exhaled breath condensate $\mathrm{pH}$ using two commercially available devices in healthy controls, asthma and COPD patients. Respir Res 2009; 10: 78.

4 de Laurentiis G, Paris D, Melck D, et al. Metabonomic analysis of exhaled breath condensate in adults by nuclear magnetic resonance spectroscopy. Eur Respir J 2008; 32: 1175-1183.

5 Carraro S, Rezzi S, Reniero F, et al. Metabolomics applied to exhaled breath condensate in childhood asthma. Am J Respir Crit Care Med 2008; 175: 986-990.

6 Montuschi P, Paris D, Melck D, et al. Metabolomic analysis by nuclear magnetic resonance spectroscopy of exhaled breath condensate in patients with cystic fibrosis. Eur Respir J 2009; 34: $63 \mathrm{~s}$.

7 Izquierdo-García JL, Peces-Barba G, Heili S, et al. Is NMR-based metabolomic analysis of exhaled breath condensate accurate? Eur Respir J 2011; 37: 468-470.

8 Hoffmeyer F, Raulf-Heimsoth M, Harth V, et al. Comparative analysis of selected exhaled breath biomarkers obtained with two different temperature-controlled devices. BMC Pulm Med 2009; 9: 48.

9 Czebe K, Barta I, Antus B, et al. Influence of condensing equipment and temperature on exhaled breath condensate $\mathrm{pH}$, total protein and leukotriene concentrations. Respir Med 2008; 102: 720-725.

10 del Campo G, Berregi I, Caracena R, et al. Quantitative determination of caffeine, formic acid, trigonelline and 5-(hydroxymethyl)furfural in soluble coffees by ${ }^{1} \mathrm{H}-\mathrm{NMR}$ spectrometry. Talanta 2010; 81: 367-371.

\section{Metabolomic signatures in nuclear magnetic resonance spectra of exhaled breath condensate identify asthma}

\section{To the Editors:}

Exhaled breath condensate (EBC) holds promise as a noninvasive method of collecting airway-lining fluid, although at an unknown dilution [1]. While metabolomic studies of EBC using nuclear magnetic resonance (NMR) spectroscopy have previously shown promise in asthma diagnosis and subtyping [2,3], a study that was recently published in the European Respiratory Journal that failed to find usable NMR signature in EBC collections from disposable systems [4]. This led the authors to conclude that NMR metabolomics lacks sufficient sensitivity for metabolic fingerprinting of EBC. Interestingly, they were able to obtain high quality results from the same samples with mass spectroscopy, which they recommended for future use. As this is a nascent and 